Title : will be set by the publisher

Editors : will be set by the publisher

EAS Publications Series, Vol. ?, 2018

\title{
METAL ENRICHMENT IN GALACTIC WINDS
}

\author{
Yohan Dubois ${ }^{1}$ and Romain Teyssier ${ }^{1}$
}

\begin{abstract}
Observations give evidences of the presence of metals in the intergalactic medium (IGM). The stars responsible for transforming hydrogen and helium into more complex atoms do not form outside the galaxies in the standard scenario of galaxy formation. Supernovaedriven winds and their associated feedback was proposed as a possible solution to explain such enrichment of the IGM. It turned out that a proper modelling of supernovae explosions within a turbulent interstellar medium (ISM) is a difficult task. Recent advances have been obtained using a multiphase approach to solve for the thermal state of the ISM, plus some additional recipes to account for the kinetic effect of supernovae on the galactic gas. We briefly describe here our implementation of supernovae feedback within the RAMSES code, and apply it to the formation and evolution of isolated galaxies of various masses and angular momenta. We have explored under what conditions a galactic wind can develop, if one considers only a quiescent mode of star formation. We have also characterized the distribution and evolution of metallicity in the gas outflow spreading in the IGM.
\end{abstract}

\section{Introduction}

Supernovae-driven winds are a key ingredient of current galaxy formation models, in order to suppress the formation of low-mass galaxies and maybe to solve the so-called "overcooling" problem in the current hierarchical scenario of structure formation in the universe. The proper modelling of galactic winds is a difficult and unavoidable task, both in semi-analytical models (Hatton et al. 2003) and in numerical simulation (Springel \& Hernquist 2003, Rasera \& Teyssier 2006) of galaxy formation. Observational evidence for galactic outflows have already been pointed out by several authors (Heckman et al 2000, Adelberger et al. 2003). They are usually associated to massive starbursts, for which very strong outflows are reported: for one solar mass of star formed in the galaxy, between 1 and 5 solar masses of gas are ejected in those winds (Martin 1999). This translates

\footnotetext{
${ }^{1}$ CEA Saclay, DSM/Dapnia/SAp, Bâtiment 709, 91191 Gif-sur-Yvette Cedex, France
} 
Title : will be set by the publisher

into a wind efficiency, usually noted $\eta_{w}$, ranging from $100 \%$ to $500 \%$. The effect of galactic outflows can also be measured in the enrichment of the intergalactic medium (IGM) as observed in absorption lines of quasars spectra (Bouché et al. 2006).

Despite the difficulties of modelling supernovae explosions within a turbulent, multiphase and magnetized ISM, understanding the physics of the resulting large scale outflows is also a challenge. Many questions arise: what are the conditions for a galactic wind to develop and escape from the galaxy potential well ? What is the mass ejection rate of such a wind ? What is the metallicity of the wind and other associated observational signatures ? As explored by Fujita et al. (2004) in the context of an isolated, pre-formed galactic disc, the ram-pressure of infalling material might be the main limiting factor for galactic winds to exist.

Our goal is here to present new simulations of galactic winds performed with the RAMSES code, using Adaptive Mesh Refinement with a state-of-the-art shockcapturing scheme (Teyssier 2002).

In this paper, we follow the approach of Springel et al. (2003), considering an isolated Navarro, Frenk \& White (1996) halo in hydrostatic equilibrium, that selfconsistently cools down and forms a centrifugally supported disc in its center. Star formation and the associated supernovae explosions proceed according to rather standard recipies.

\section{Numerical methods}

We have considered that initially gas and dark matter follow the same NFW mass density profile, with $15 \%$ of the total mass in baryons in hydrostatic equilibrium. The concentration parameter was set to $c=10$, independant of the halo mass. The initial angular momentum is given by the fitting formula $j(r)=j_{\max } M(r) / M_{\text {vir }}$ of Bullock et al. (2001), normalized to the halo spin parameter $\lambda=J|E|^{1 / 2} / G M_{v i r}^{5 / 2}$, for which we have considered two cases: $\lambda=0.04$ and $\lambda=0.1$.

The gas is radiatively cooled using a metallicity-dependant cooling function (Courty \& Teyssier, in prep.), since metal enrichment by supernovae feedback is self-consistently simulated in this study. In order to take into account the thermal feedback of supernovae explosions, we have implemented a simplified form of the multiphase model of Springel \& Hernquist (2003), using a polytropic equation of state in the regions of star formation $\left(n_{0}>0.1 \mathrm{~cm}^{-3}\right)$ and where the temperature is fixed to the polytropic equilibrium temperature. In these regions a fraction of gas is converted into star particles using a Schmidt law, with a time scale parametrized by $t_{*}=t_{0}\left(n_{H} / n_{0}\right)^{-1 / 2}$. We chose $t_{0}=3 \mathrm{Gyr}$ or $8 \mathrm{Gyr}$ in our simulations, resulting in different overall star formation efficiencies (see Rasera \& Teyssier for details).

Kinetic feedback due to supernovae explosions that we have implemented in RAMSES is the key process of this study. For each star particle formed, we create "debris particles", containing both supernovae ejecta and surrounding gas entrained by the blast wave. The velocity of the "debris particles" is computed according to a local Sedov solution. Debris propagate freely as collisionless particles 
over a distance corresponding to a blast wave radius of 2 cells. At this moment, debris release their mass, metal content, momentum and energy to the gas cell they have reached.

We have performed isolated galaxy simulations with two different virial masses $10^{10} \mathrm{M}_{\odot}$ and $10^{11} \mathrm{M}_{\odot}$ in a box of $6 R_{v i r}$ and over 6 Gyr. The coarse grid of those simulations has $128^{3}$ cells and the maximum resolution is about $150 \mathrm{pc}$.

\section{A toy model for galactic winds}
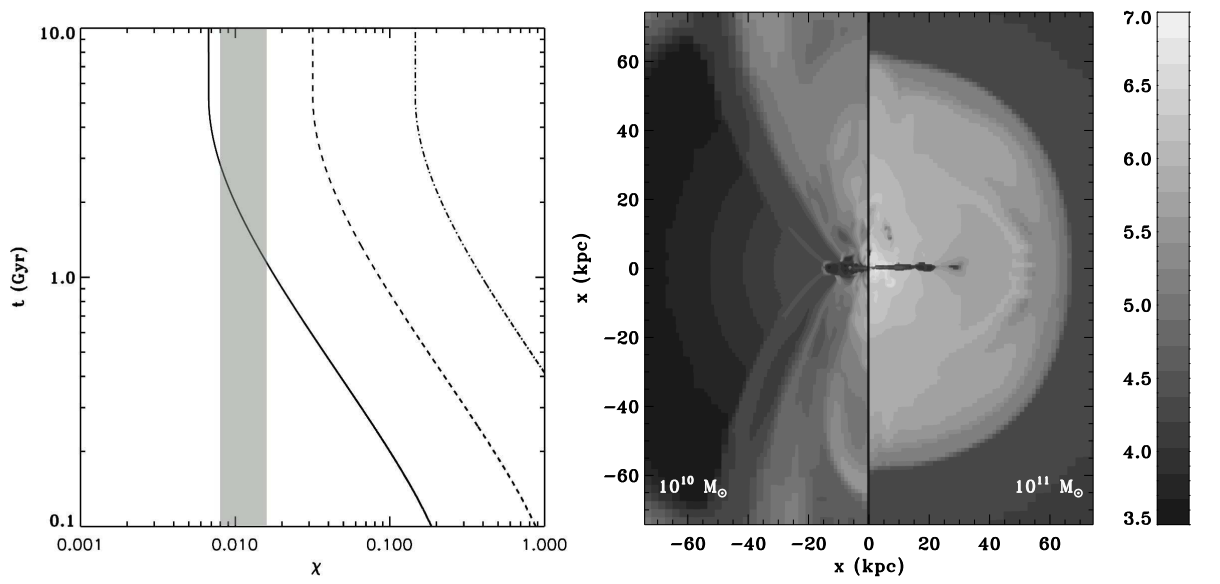

Fig. 1. Left: Wind break-out epoch as a function of hydrodynamical efficiency $\chi$ for three different halo masses: $10^{10} \mathrm{M}_{\odot}, 10^{11} \mathrm{M}_{\odot}, 10^{12} \mathrm{M}_{\odot}$ (from left to right). The shaded area corresponds to the allowed range, as demonstrated by our numerical simulations. Right: Comparison of the temperature map obtained at $t=6$ Gyr for 2 different simulations, with a galactic wind breaking out of the low mass halo (left), and a galactic fountain remaining close to the disc in the high mass case (right).

Following Fujita et al. (2004), we anticipate that the infall of gas from the halo will be the key process in the success or the failure of galactic outflows. Gas infall fuels the star forming disc with fresh gas, and therefore controls the supernovae explosion rate in the galaxy but it is also the source of ram pressure that can confine any outflowing material from the disc. We propose here a very simple model in which the total gas mass evolves according to the following "open box solution"

$$
M_{g}(t)=\int_{0}^{t} \exp \left(\frac{u-t}{t_{*}}\right) \dot{M}_{a c c}(u) \mathrm{d} u, \quad \text { and } \quad \dot{M}_{*}(t)=\frac{M_{g}(t)}{t_{*}} .
$$

The accretion rate $\dot{M}_{a c c}$ is computed assuming that each shell of our NFW halo free-falls down to the center, where the disc has formed. We then compare the 
Title : will be set by the publisher

supernovae luminosity in the disc to the accretion luminosity of infalling gas

$$
L_{w}(t)=\chi \dot{M}_{*}(t) \eta_{s n} \frac{E_{S N}}{M_{S N}}, \quad \text { versus } \quad L_{a c c}(t)=\frac{1}{2} \dot{M}_{a c c} v_{\infty}^{2}
$$

where $v_{\infty}$ is the terminal velocity of free-falling gas shells, computed using the NFW mass profile. The key parameter of the model, $\chi$, is called the "hydrodynamical efficiency" and refers to the conversion efficiency of supernovae energy into galactic wind energy. $\eta_{S N} \simeq 10 \%$ is the mass fraction of stars that explode into type II supernovae, according to standard stellar initial mass function, $E_{S N} \simeq 10^{51}$ erg is the typical energy produced by one single supernova, and $M_{S N} \simeq 10 \mathrm{M} \odot$ is its typical progenitor mass. A part of the energy of supernovae explosions creates heating and turbulence in the multiphase ISM. Since this energy dissipated in the ISM does not contribute in the global outflow, the hydrodynamical efficiency is likely to be small. The fraction of energy that manage to escape from the dense gaseous disc will depend on the disc characteristic (thickness, size, gas content). In our simulations, these properties will be specified by the the spin parameter of the halo and by the star formation time scale of the Schmidt law. In Figure 1 the wind break-out epoch (i.e. when the wind luminosity exceeds the accretion luminosity) is shown for various halo masses, as a function of the unknown parameter $\chi$. One clearly sees that the smaller the hydrodynamical efficiency, the later the wind will blow out of the disc. More importantely, for each halo mass, a minimum efficiency is required in order for a wind to appear: $0.7 \%$ (resp. $3 \%$ and $15 \%$ ) for a $10^{10} \mathrm{M}_{\odot}$ halo (resp. $10^{11}$ and $10^{12}$ ).

\section{Simulation results}

Our various simulations draw a similar qualitative picture: a cold centrifugally supported disc form at the halo center from the inside out, whose size depends mainly on the halo spin parameter. The star formation rate in the galaxy rises sharply (see Figure 2) and hot supernovae bubbles start to break out of the disc. After roughly 1 to 3 Gyr, either a galactic wind develops (for the low mass halo) or a galactic fountain sets in (for the high mass halo). Figure 1 shows a map of the gas temperature obtained after 6 Gyr in both cases. One clearly sees a noozle-like structure escaping from a small, thick disc in the $10^{10} \mathrm{M} \odot$ case, while only hot plumes and cold clumps are seen in the $10^{11} \mathrm{M} \odot$ case, oscillating close to a large thin disc, as they are confined by a hot, ram pressure driven atmosphere.

In figure 2 we have plotted the mass flux flowing out of the disc, computed in a spherical shell of size $r=\left[5 r_{s} ; 7 r_{s}\right]$, in the $10^{10} \mathrm{M}_{\odot}$ case only, for various values of $\lambda$ and $t_{0}$. The wind break-out epoch can be determined with great accuracy: it appears as a sharp rise in the mass outflow rate curve. Comparing the apparition time of the wind to the SFR, we can asses that a strong SFR at early epoch of galaxy formation creates a young wind that carries out a big quantity of the hot gas of the halo during the first blow. Using our toy model, we can determine the corresponding hydrodynamical efficiency, which, depending on the simulation parameters, varies between 0.8 to $2 \%$. Injecting these values in the toy model for a 
$10^{11} \mathrm{M}_{\odot}$ halo, we predict that no wind can break-out in this case. Our numerical simulations do confirm this, as they show only a galactic fountain with no gas leaving the halo potential well.

When the galactic wind is fully developped, it shows a typical nozzle shape, and the mass outflow rate reaches its asymptotic value, around $0.01 \mathrm{M}_{\odot} / \mathrm{yr}$. One can then compute the wind efficiency, defined as $\eta_{w}=\dot{M}_{w} / \dot{M}_{*}$, which ranges from 10 to $20 \%$, an order of magnitude below what is expected from Lyman Break galaxies observations (Martin 1999).
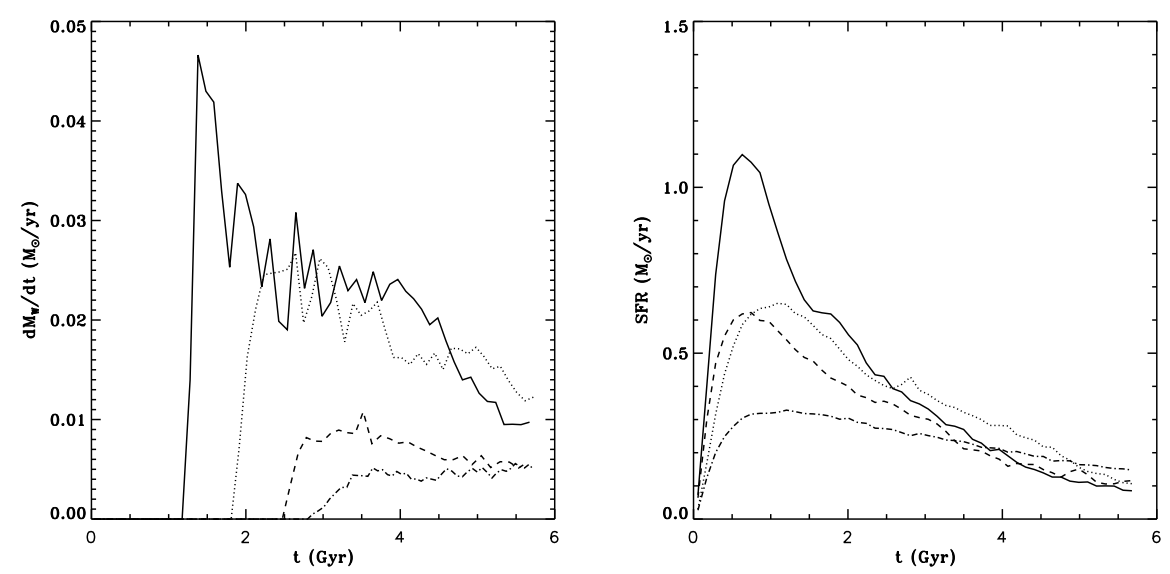

Fig. 2. Flux of mass (left) outflowing of the $10^{10} \mathrm{M}_{\odot}$ halos calculated between $r=$ $\left[5 r_{s} ; 7 r_{s}\right]$ and SFR (right) for the $\lambda=0.04$ and $t_{0}=3 \mathrm{Gyr}$ (solid line), $\lambda=0.04$ and $t_{0}=8 \mathrm{Gyr}$ (dotted line), $\lambda=0.1$ and $t_{0}=3 \mathrm{Gyr}$ (dashed line) and $\lambda=0.1$ and $t_{0}=8 \mathrm{Gyr}$ (dash-dotted line).

Figure 3 shows the mean metallicity projected along the rotation axis of the disc for different times of the galaxy evolution (3 Gyr, 4.5 Gyr, 6 Gyr). The $10^{10} \mathrm{M}_{\odot}$ halo begins with a moderately enriched wind $\left(Z<0.3 \mathrm{Z}_{\odot}\right)$. The wind propagates in the IGM with a metallicity growing up to about $\mathrm{Z}_{\odot}$ at $6 \mathrm{Gyr}$, while the disc barely reaches $0.4 \mathrm{Z}_{\odot}$. The behavior of the $10^{11} \mathrm{M}_{\odot}$ halo is quite different because the gas flowing out of the disc falls back. There are two consequences: the gas in the galactic fountain remains at a constant metallicity around $0.2 \mathrm{Z}_{\odot}$ and the disc progressively accumulates metals up to $\mathrm{Z}_{\odot}$. The galactic disc can be seen as a closed system where no gas is lost in the galactic wind.

\section{Conclusion}

Using a quiescent model of star formation in isolated galaxies, self-consistently simulated from a cooling NFW halo, we have studied the conditions for a galactic wind to break-out of the dark matter halo potential well. Our simulations have shown that no wind can form in halo of mass greater than $10^{11} \mathrm{M}_{\odot}$, even for 

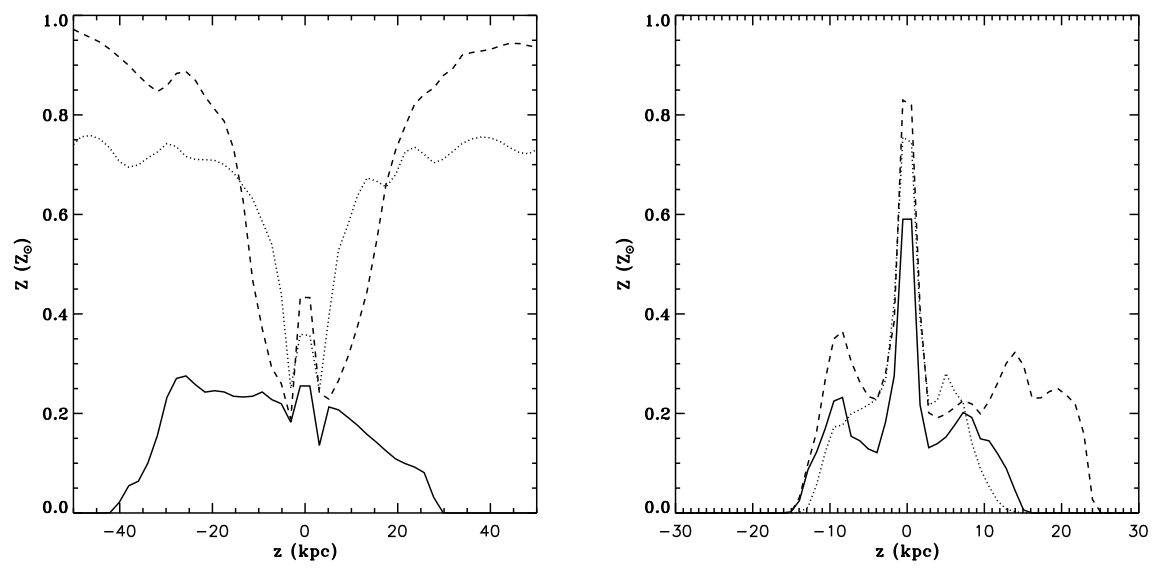

Fig. 3. Mean metallicity of the gas as a function of the projected height for the $10^{10} \mathrm{M}_{\odot}$ halo (left) and the $10^{11} \mathrm{M}_{\odot}$ halo (right) at different times: $t=3 \mathrm{Gyr}$ (solid line), $t=$ 4.5 Gyr (dotted line) and $t=6 \mathrm{Gyr}$ (dashed line) for $\lambda=0.1$ and $t_{*}=8 \mathrm{Gyr}$.

our most favorable couple of halo parameters $\left(\lambda=0.04, t_{0}=3 \mathrm{Gyr}\right)$. Using a simple toy model, we understand this failure as due to the ram pressure of infalling material confining the outflowing wind. Using a more realistic cosmological setting may result into non-spherical accretion flows, and therefore to a less stringent criterion for a wind to break-out. A proper modelling of starburst (yet to be invented) might also provide an easier route for increasing the feedback efficiency of supernovae-driven outflows.

\section{References}

Adelberger, K. L., Steidel, C. C., Shapley, A. E., \& Pettini, M. 2003, ApJ, 584, 45

Bouché, N., Lehnert, M. D., \& Péroux, C. 2006, MNRAS, 367, L16

Bullock, J. S., Dekel, A., Kollat, T. S., et al. 2001, ApJ, 555, 240

Fujita, A., Mac Low, M.-M., Ferrara, A., \& Meiksin, A. 2004, ApJ, 613,159

Hatton, S., Devriendt, J. E. G., Ninin, S., et al. 2003a, MNRAS, 343, 75

Heckman, T. M., Lehnert, M. D., Strickland, D. K., \& Armus, L. 2000, ApJS, 129, 493

Martin C.L., 1999, ApJ, 513, 156

Navarro, J. F., Frenk, C. S., \& White, S. D. M. 1996, ApJ, 462, 563

Rasera, Y. \& Teyssier, R. 2006, A\&A, 445, 1

Springel, V. \& Hernquist, L. 2003, MNRAS, 339, 289

Teyssier, R. 2002, A\&A, 385, 337 